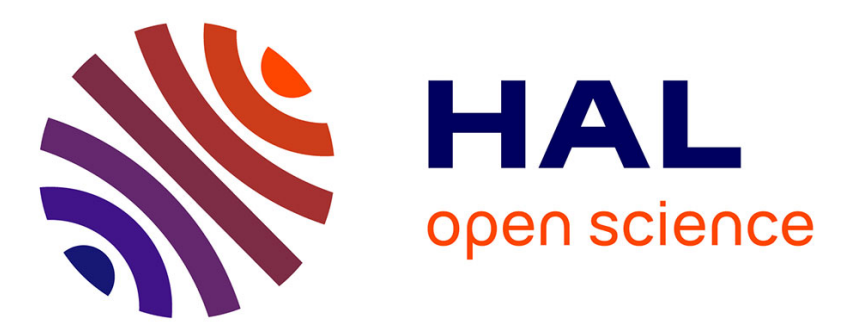

\title{
Comment on "Effect of the flow state on streaming current" by Osamu Kuwano, Masao Nakatani, and Shingo Yoshida
}

\author{
André Revil
}

\section{- To cite this version:}

André Revil. Comment on "Effect of the flow state on streaming current" by Osamu Kuwano, Masao Nakatani, and Shingo Yoshida. Geophysical Research Letters, 2007, 34, pp.L09311. 10.1029/2006GL028806 . insu-00404730

HAL Id: insu-00404730

https://hal-insu.archives-ouvertes.fr/insu-00404730

Submitted on 2 Jun 2021

HAL is a multi-disciplinary open access archive for the deposit and dissemination of scientific research documents, whether they are published or not. The documents may come from teaching and research institutions in France or abroad, or from public or private research centers.
L'archive ouverte pluridisciplinaire HAL, est destinée au dépôt et à la diffusion de documents scientifiques de niveau recherche, publiés ou non, émanant des établissements d'enseignement et de recherche français ou étrangers, des laboratoires publics ou privés.

$$
\text { Copyright }
$$




\title{
Comment on "Effect of the flow state on streaming current" by Osamu Kuwano, Masao Nakatani, and Shingo Yoshida
}

\author{
André Revil ${ }^{1}$ \\ Received 17 November 2006; revised 12 February 2007; accepted 28 February 2007; published 9 May 2007.
}

Citation: Revil, A. (2007), Comment on "Effect of the flow state on streaming current' by Osamu Kuwano, Masao Nakatani, and Shingo Yoshida, Geophys. Res. Lett., 34, L09311, doi:10.1029/2006GL028806.

[1] Kuwano et al. [2006] (hereinafter referred to as KNY06) investigated recently how the apparent permeability and the apparent streaming current coupling coefficient depend on the Reynolds number. This is a very interesting problem with several potential applications in geophysics like the detection of hydromechanical disturbances in active volcanoes. However, this problem has received very limited attention so far. KNY06 realized experiments showing a decrease of the streaming current coupling coefficient $L$ and permeability $k$ of glass bead packs with the Reynolds number Re. They also noted qualitatively that $L$ decreases less than $k$ with Re and suggested that this difference could be related to the increase in the efficiency of charge transport in the vicinity of the surface of the minerals when the Reynolds number increases. This effect reflects indeed a change in the shape of the velocity profile of the water in the pores when the Reynolds number increases. However, KNY06 did not use an appropriate definition of the Reynolds number and we show below that their values of the Reynolds numbers are overestimated by a factor $\sim 24$. KNY06 did not also recognize the importance of the transition between the viscous- and the inertial-laminar flow regime in explaining quantitatively their experimental results. We show that the dependence of the apparent permeability with the Reynolds number can be quantitatively analyzed from the work published by Teng and Zhao [2000]. The dependence of the streaming current coupling coefficient with the Reynolds number has also been investigated very recently by Watanabe and Katagishi [2006] and Crespy et al. [2007].

[2] In this comment, I will use a more appropriate expression of the Reynolds number. This expression implies values by one order of magnitude smaller than those used by KNY06 and I provide a model and quantitative expressions regarding how to determine the influence of the Reynolds number upon the permeability and the streaming current coupling coefficient for porous media. These equations improve strongly the interpretation of the very interesting experimental data obtained by KNY06 by explaining quantitatively their results inside a theoretical framework.

\footnotetext{
${ }^{1}$ Centre Européen de Recherche et d'Enseignement de Géosciences de l'Environnement, CNRS, Université Paul Cézanne, Aix-en-Provence, France.
}

Copyright 2007 by the American Geophysical Union. 0094-8276/07/2006GL028806
[3] Teng and Zhao [2000] derived recently a generalized Darcy equation by volume-averaging the local NavierStokes momentum equation over a representative elementary volume of a porous material. Their final result is,

$$
\rho_{f} \frac{d \mathbf{U}}{d t}+\frac{1+\operatorname{Re}}{k_{0}} \eta_{f} \mathbf{U}=-\nabla p+\mathbf{F},
$$

where $\eta_{f}$ (in Pa s) is the dynamic viscosity of water, $p$ (in Pa) is the pore fluid pressure, $\mathbf{U}$ is the seepage (Darcy) velocity (in $\mathrm{m} \mathrm{s}^{-1}$ ), $t$ is time (in s), $k_{0}$ (in $\mathrm{m}^{2}$ ) is the intrinsic permeability of the porous material in the viscous-laminar flow regime characterized by low values of the Reynolds number $\mathbf{R e}$, and $\mathbf{F}$ is a macroscopic body force. The Reynolds number is a key-dimensionless number that expresses the ratio of inertial to viscous forces in the Navier-Stokes equation [e.g., Batchelor, 1972]. For a capillary of radius $R, U$ being the strength of the seepage velocity, the Reynolds number is a well-defined parameter given by [e.g., Batchelor, 1972],

$$
\operatorname{Re}=\frac{\rho_{f} U R}{\eta_{f}},
$$

where $\rho_{f}$ is the mass density of water. In a porous material, the radius of the capillary should be replaced by a corresponding length scale of the porous material. We cannot replace directly the radius of the capillary by the radius of the grain because these two quantities have a very different hydrodynamic meaning. I propose that the Reynolds number of a porous material is defined by,

$$
\operatorname{Re}=\frac{\rho_{f} U \Lambda}{\eta_{f}},
$$

where $\Lambda$ is the characteristic length of the flow (for capillaries $\Lambda=R$ where $R$ is the radius of the capillary) introduced by Kostek et al. [1992]. We consider from now steady-state flow like done in KNY06. The experiment can be done in a tube with a hydraulic head $h$ and the porous medium can be packed in a vertical cylinder of length $L$ as done by Crespy et al. [2007]. In this case, the pressure gradient is $(h / L) \hat{\mathbf{z}}$ where $\hat{\mathbf{z}}$ is the vertical unit vector directed upward. Taking equation (1) in steady-state conditions and neglecting the body force, the Darcy velocity can be expressed in an apparent Darcy's law $\mathbf{U}=-\left(k \rho_{f} g / \eta_{f}\right)(h / L) \hat{\mathbf{z}}$ where $g=9.81 \mathrm{~m}^{2} \mathrm{~s}^{-1}$ (acceleration of the gravity) and $k$ is an apparent permeability that is related to the Reynolds number by,

$$
k / k_{0}=1 /(1+\mathrm{Re})
$$




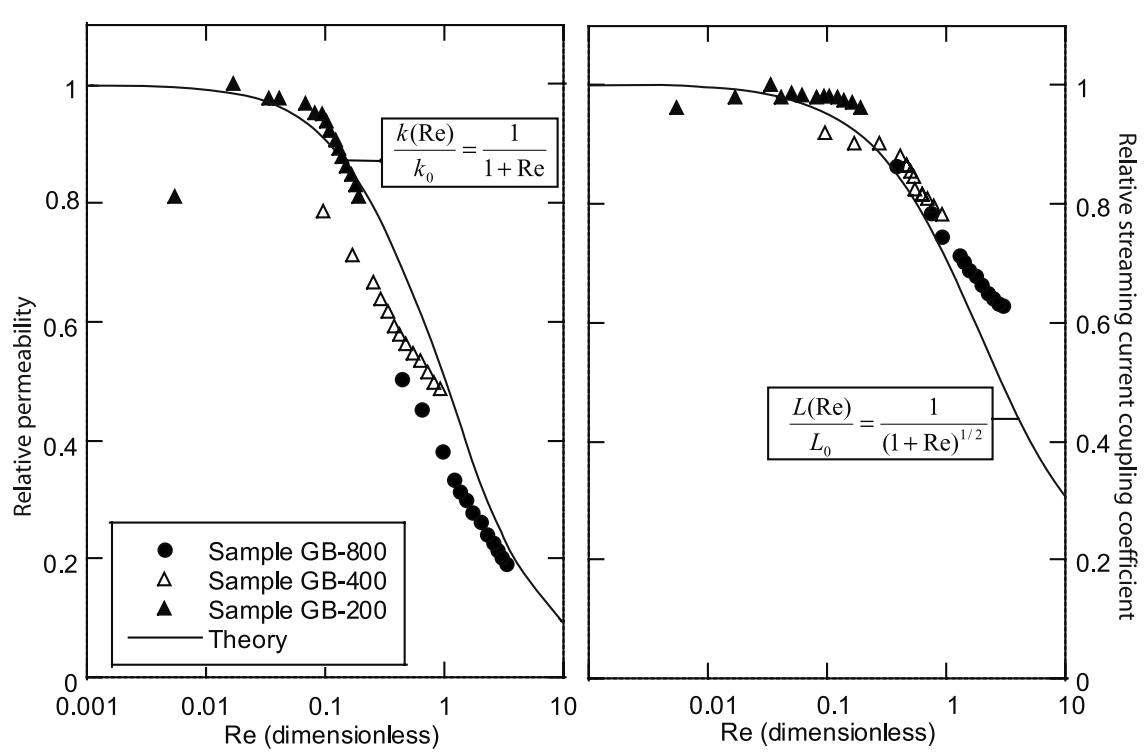

Figure 1. Comparison between the data of KNY06 (plotted with the new estimates of the Reynolds number, see main text) and the model discussed in this comment for macro-porous media.

[4] We can now take equation (3) and we can replace $U$ by $\left(k \rho_{f} g / \eta_{f}\right)(h / L)$. The Reynolds number is given by,

$$
\operatorname{Re}=\frac{\rho_{f}^{2} g k_{0} \Lambda}{\eta_{f}^{2}(1+\mathrm{Re})} \frac{h}{L}
$$

[5] For a granular medium with grain a diameter $d_{0}$ (in $\mathrm{m})$, the length scale $\Lambda$ is related to $d_{0}$ by $\Lambda=d_{0} /[2 m(F-1)]$ and the intrinsic permeability is related to $d_{0}$ by $k_{0}=d_{0}^{2} /[\alpha$ $\left.F(F-1)^{2}\right]$ where $\alpha$ is a numerical constant in the range $8-53$ and where $F=\phi^{-m}$ is the electrical formation factor and $m$ is the cementation exponent [Revil, 2002]. From these relationships and equation (5), the Reynolds number is the solution of the following equation,

$$
\operatorname{Re}^{2}+\operatorname{Re}-\frac{\rho_{f}^{2} g}{2 m \alpha \eta_{f}^{2}} \frac{d_{0}^{3}}{F(F-1)^{3}}\left(\frac{h}{L}\right)=0 .
$$

[6] The positive root of equation (6) is,

$$
\begin{gathered}
\operatorname{Re}=\frac{1}{2}(\sqrt{1+c}-1), \\
c=\frac{\vartheta \rho_{f}^{2} g}{\eta_{f}^{2}} \frac{d_{0}^{3}}{F(F-1)^{3}}\left(\frac{h}{L}\right),
\end{gathered}
$$

where $\vartheta \approx 2.25 \times 10^{-3}$ is a numerical factor determined from average values for $m$ and $\alpha$. Equation (7) has been used successfully by Crespy et al. [2007]. A comparison between this formula and the one used by KNY06 shows that the estimates of the Reynolds numbers obtained by KNY06 are overestimated by a factor $\sim 24$. The discrepancy is even worst if I use the value of the formation factor provided by KNY06. A better estimate of the formation factor is discussed below.
[7] For the dependence of the streaming current coupling coefficient with Re, Crespy et al. [2007] derived the following equation,

$$
L(\operatorname{Re})=L_{0}(1+\mathrm{Re})^{-n} .
$$

with $n=1$.

[8] To quantitatively apply the previous model to the data of KNY06, we need an estimate of the electrical formation factor. KNY06 used a linear model that relates the electrical conductivity of the packs to the electrical conductivity of the electrolyte. This is a very simplified model at low pore water conductivities [see Crespy et al., 2007, and references therein]. The formation factor estimated by KNY06 $(F=18)$ seems to be severely overestimated. Archie's law with a porosity in the range $0.35-0.40$ (typically the porosity of a random pack of spherical grains), and a cementation exponent $m$ in the range $1.3-1.5$, yield a formation factor below 10. Measurements carried out by Crespy et al. [2007] indicates that $F=3.3 \pm 0.1$ for glass bead packs and we believe that this value is much more representative than the value provided by KNY06. Considering this value of the formation factor, we have re-analyzed the data of KNY06. The results are displayed on Figure 1 and are consistent with the model discussed above but with $n=1 / 2$ in equation (9). The theoretical reason for this exponent will be explored in a forthcoming contribution.

[9] We conclude that the scaling of the permeability and the streaming current coupling coefficient with the Reynolds number can be quantitatively analyzed with simple functional dependence on the Reynolds number.

[10] Acknowledgments. I thank S. Byrdina and N. Linde for fruitful discussions and the two referees for constructive comments.

\section{References}

Batchelor, G. K. (1972), An Introduction to Fluid Dynamics, Cambridge Univ. Press, New York.

Crespy, A., A. Bolève, and A. Revil (2007), Influence of the Dukhin and Reynolds numbers on the apparent zeta potential of granular media, J. Colloid Interface Sci., 305, 188-194. 
Kostek, S., L. M. Schwartz, and D. L. Johnson (1992), Fluid permeability in porous media: Comparison of electrical estimates with hydrodynamical calculations, Phys. Rev. B, 45, 186-195.

Kuwano, O., M. Nakatani, and S. Yoshida (2006), Effect of the flow state on streaming current, Geophys. Res. Lett., 33, L21309, doi:10.1029/ 2006GL027712.

Revil, A. (2002), The hydroelectric problem of porous rocks: Thermodynamic approach and introduction of a percolation threshold, Geophys. J. Int., 151(3), 944-949.

Teng, H., and T. S. Zhao (2000), An extension of Darcy's law to non-Stokes flow in porous media, Chem. Eng. Sci., 55, 2727-2735.
Watanabe, T., and Y. Katagishi (2006), Deviation of linear relation between streaming potential and pore fluid pressure difference in granular material at relatively high Reynolds numbers, Earth Planets Space, 58(8), $1045-$ 1051 .

A. Revil, Centre Européen de Recherche et d'Enseignement de Géosciences de l'Environnement, CNRS, Université Paul Cézanne, Aix-en-Provence, France. (revil@cerege.fr) 